\title{
Propiedades termo-mecánicas del Polipropileno: Efectos durante el reprocesamiento
}

\section{Thermo-mechanical properties of Polypropylene: Effects during reprocessing}

\section{Caicedo Carolina}

Centro Nacional de Asistencia Técnica a la Industria (ASTIN)

Servicio Nacional de Aprendizaje-SENA, Colombia

Correo: carolina.caicedo@gmail.com

\section{Crespo-Delgado Lina Marcela}

Centro Nacional de Asistencia Técnica a la Industria (ASTIN)

Servicio Nacional de Aprendizaje-SENA, Colombia

Correo: Imcrespo3@misena.edu.co

\author{
De La Cruz-Rodríguez Hever \\ Centro Nacional de Asistencia Técnica a la Industria (ASTIN) \\ Servicio Nacional de Aprendizaje-SENA, Colombia \\ Correo: hdelacruz@misena.edu.co \\ Álvarez-Jaramillo Norman Andrés \\ Centro Nacional de Asistencia Técnica a la Industria (ASTIN) \\ Servicio Nacional de Aprendizaje-SENA, Colombia \\ Correo: naalvarez208@misena.edu.co
}

\section{Resumen}

En este trabajo se llevó a cabo la transformación del copolímero al azar a base de polipropileno mediante la técnica de inyección. Se establecieron las variables que corresponden a temperatura, presión, velocidad y tiempo de residencia para el proceso del material virgen. Posteriormente, se obtuvieron cinco generaciones bajo las mismas condiciones de proceso con el objeto de estudiar las propiedades térmicas y mecánicas del mismo, que permitió relacionar los cambios debidos a la termoxidación del material. Los resultados de los análisis correspondientes a la caracterización mecánica presentaron disminución hasta $6.0 \%$ para flexión y $8.4 \%$ para tracción. Por otra parte, los resultados térmicos mostraron $13.6 \%$ de pérdida en la quinta generación, respecto al material virgen en el experimento de procesamiento con la inyectora, mientras que para el proceso de simulación térmica la pérdida fue de 3.8\%. Esta simulación consideró como parámetros: la temperatura máxima, el tiempo de residencia y el número de ciclos de proceso. Este análisis térmico permitió cuantificar los efectos asociados a la fricción como resultante de los esfuerzos mecánicos de corte y compresión.

Descriptores: Polipropileno, inyección, análisis térmico, reproceso, propiedades mecánicas.

\begin{abstract}
In this work a transformation of random polypropylene copolymer by injection technique was carried out. For the virgin material process the corresponding variables such as temperature, pressure, velocity and residence time were established. In order to study the thermal and mechanical properties of this system. Five more generations were obtained, which allowed us to correlate the changes caused by the material thermal oxidation. The results of mechanical characterization corresponding to the analysis presented to decrease in $6.0 \%$, for bend and $8.4 \%$ for traction. On the other hand, the thermal results showed a $13.6 \%$ loss on the fifth generation regarding the virgin material on the injection experiment; meanwhile for the thermal simulation this loss was minimal with a $3.8 \%$. For the simulation three parameters were considered: maximum temperature, residence time and the number of cycles in the process. The thermal analysis allowed us to quantify the friction associated effects as a result of the mechanical shear and compression.
\end{abstract}

Keywords: Polypropylene, injection, thermal analysis, reprocess, mechanical properties. 


\section{INTRODUCCIÓN}

El Polipropileno (PP) es ampliamente utilizado para la producción de plásticos moldeados debido a la excelente combinación de propiedades que presenta como peso ligero y resistencia al impacto (Harutun, 2003). Hoy en día, este material presenta una demanda global de alrededor de 55 millones de toneladas al año (Market Study, 2013). Con lo anterior, los polímeros han logrado desplazar a los materiales metálicos y cerámicos (Brenner, 2000). Asimismo, se ha logrado convertir en un fenómeno para la producción de múltiples aplicaciones mediante el uso de técnicas convencionales como es el caso de la Extrusión, Inyección y Termoconformado para el desarrollo de recipientes rígidos de embalaje, electrodomésticos, herramientas de mano, películas elásticas, fibras y telas, piezas para vehículos, materiales del sector médico y farmacéutico, entre otros (Hoffman, 2002). Por lo anterior, muchas compañías han ultimado la fase de construcción de plantas para la producción de PP. El copolímero al azar a base de polipropileno (polypropylene random copolymer, PP) a diferencia del homopolímero presenta una buena rigidez, una importante ductilidad, un aceptable límite elástico y una excelente resistencia química a un precio relativamente bajo (Papageorgioua et al., 2012). El contenido de etileno en PP se caracteriza por su baja cristalinidad y propiedades elastoméricas, por lo cual, estos materiales suelen emplearse como mejoradores de impacto en mezcla con otros materiales. Los proyectos de investigación aplicada hoy en día, tienden a realizar modificaciones sobre matrices de PP por medio del desarrollo de materiales compuestos utilizando partículas a nano- y micro-escala (Thakur et al., 2014), fibras (Bledzki et al., 2015) y mediante mezclas o aleaciones con otros polímeros (Kang et al., 2015). Estudios recientes muestran la importancia de caracterizar estos materiales con diferentes carreras de proceso debido a que muestran la importancia de funcionalización sobre la matriz (Bahlouli et al., 2015; Da Costa et al., 2007). En la evaluación de propiedades fisicoquímicas se consideran diversas técnicas como por ejemplo, la termogravimetría, con la que se identifica la temperatura de degradación y estabilidad térmica, la calorimetría diferencial de barrido indica los cambios de fase asociados al material. Esta última permite realizar estudios dinámicos a través de los diferentes tratamientos y ciclos de proceso. Por otro lado, es posible determinar el comportamiento mecánico como la resistencia a la tensión y módulo de Young o elástico en función a la deformación mediante ensayo de tracción y flexión, respectivamente. Diversos estudios presentan como parámetro esencial la determinación del índice de fluidez, viscosidad y densidad debido a que permiten identificar las características reológicas con el fin de evidenciar efectos de reproceso y optimizar el procesamiento de los plásticos (González et al., 1998). En general, un material polimérico puede transformarse por acción de la temperatura y cada ciclo que atraviesa se encuentra representado en un historial térmico. Es imperativo realizar una adecuada caracterización fisicoquímica a cada generación del material, que permita correlacionar las propiedades que están íntimamente ligadas a la estructura molecular o microestructura del polímero. Algunos trabajos relacionados con materiales poliméricos reciclados justifican la disminución en el peso molecular del polímero o escisiones de la cadena a causa de los múltiples procesos termomecánicos (Hermanová et al., 2009). A pesar de que el polipropileno es ampliamente comercializado, se sigue estudiando con el fin de optimizar y potencializar su amplio rango de aplicaciones. En la actualidad, la mediana y pequeña industria del plástico presenta desconocimiento frente a las propiedades fisicoquímicas del polipropileno de alto impacto (copolímero) reprocesado, lo anterior, limita el aprovechamiento del material y ocasiona continuos reaustes en los parámetros durante el proceso. En este trabajo se analiza la influencia de la temperatura sobre el polipropileno con múltiples ciclos de inyección procesado bajo las mismas condiciones, determinando el índice de desempeño termo-mecánico del mismo.

\section{Parte experimental}

\section{MATERIAlES}

Copolímero al azar a base de polipropileno 02R01CA-1 producido por Essentia en forma de gránulos, presenta un índice de fluidez de $1.6 \mathrm{~g} / 10 \mathrm{~min}$. La metodología del proceso se resume en la figura 1 y las etapas sucesivas se detallan a continuación.

\section{CICLOS DE INYECCIÓN Y MOLIENDA}

El PP se moldeó cinco veces por inyección con una máquina inyectora DEMAG de 150 toneladas, modelo 1991, hidráulica y de válvulas insertables (capacidad máxima de plastificación: 230 g, distancia entre columnas: $460 \mathrm{~mm}$, distancias de cierre: mínima $220 \mathrm{~mm}$, máxima $450 \mathrm{~mm}$, carrera máxima de apertura: $450 \mathrm{~mm}$ ). El perfil de temperatura fue de $210^{\circ} \mathrm{C}-220^{\circ} \mathrm{C}-230^{\circ} \mathrm{C}$ y $240^{\circ} \mathrm{C}$ para la boquilla. La presión de inyección se mantuvo constante a 75.6 bares; la temperatura del molde se fijó en $45^{\circ} \mathrm{C}$ y se aplicó una velocidad de inyección constante de $45 \mathrm{~cm}^{3} . \mathrm{s}^{-1}$. Los parámetros de inyección 
son los mismos para cada ciclo. Las probetas obtenidas fueron tipo $1 \mathrm{~b}$ "hueso" de acuerdo con la norma ISO 527-2. Las muestras se diferencian por el siguiente símbolo PP con el número de ciclo de proceso como subíndice, por ejemplo, $\mathrm{PP}_{2}$ es un $\mathrm{PP}$ reprocesado por segunda vez. Finalmente, los gránulos se obtuvieron con un diámetro de $8 \mathrm{~mm}$ aproximadamente, en un molino de cuchillas Rotrogram Mould-tek a 1745 rpm, este material molido se utilizó en la etapa 3 del método de procesamiento mostrado en la figura 1. Finalmente, se obtienen 5 muestras de $\mathrm{PP}_{1}$ en la primera inyección y 4 reprocesos hasta $\mathrm{PP}_{5}$.

\section{SimulaCión tÉRMICA}

El material virgen $\left(\mathrm{PP}_{0}\right)$ se sometió a varios ciclos de calentamiento en un horno convencional a la temperatura máxima de proceso por el tiempo de residencia $\left(t_{r}\right)$ obtenido en el experimento. El $t_{r}(1)$ se calculó estimando las siguientes variables: peso del material inyectado $\left(w_{i}\right)$, tiempo de ciclo $\left(t_{c}\right)$ y peso del material en el barril $w_{t}(2)$ (Muccio, 1994).

$t r=\frac{w_{t}}{w_{i}} \cdot t_{c} \quad w g \quad V$

$w_{t}(g)=\rho_{m} \cdot V_{t}$

La ecuación 2, sugiere determinar la gravedad específica del material en estado fundido $\rho_{m}$ y estimar el volumen del material en el barril $\left(V_{t}\right)$ aplicando la siguiente expresión matemática

$V_{t}=\frac{\pi\left(D_{b}^{2}-D_{r}^{2}\right) L_{b}}{2}$

donde

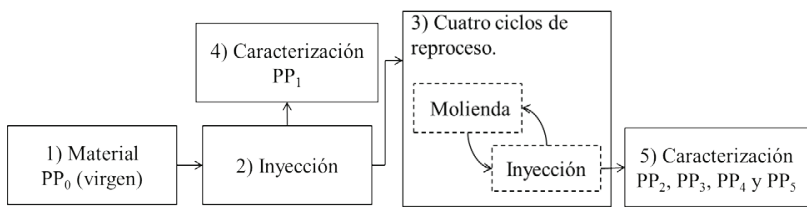

Figura 1. Etapas del método de procesamiento por molienda e inyección
$D_{b}=$ diámetro del barril

$L_{b}=$ longitud del barril y

$D_{r}=$ diámetro de raíz del tornillo, como lo muestra la figura 2.

Después de obtener las diferentes generaciones por simulación térmica (solo calentamiento) del material $\left(\mathrm{PP}_{1 \mathrm{~s}}, \mathrm{PP}_{2 \mathrm{~s}}, \mathrm{PP}_{3 \mathrm{~s}}, \mathrm{PP}_{4 \mathrm{~s}}\right.$ y $\left.\mathrm{PP}_{5 \mathrm{~s}}\right)$ se procedieron a analizar las propiedades térmicas con el fin de comparar los valores correspondientes a temperatura de degradación y temperatura de fusión mediante TGA y DSC a las condiciones que se mencionan a continuación.

\section{CARACTERIZACIÓN}

Termogravimetría (TGA) y Calorimetría Diferencial DE BARRIDO (DSC)

Las propiedades térmicas se determinaron en un analizador termogravimétrico TGA/DSC 2 STAR System, Mettler Toledo. Las muestras $(10 \pm 0.5 \mathrm{mg})$ se colocaron en crisoles de alúmina en un rango de temperatura entre $25^{\circ} \mathrm{C}$ a $400^{\circ} \mathrm{C}$ bajo atmosfera de nitrógeno $\left(50 \mathrm{~cm}^{3} /\right.$ min). Se trabajó según las normas ASTM E1131-98 y ASTM D3418-12, respectivamente. La determinación del índice de desempeño térmico (I.D.T) para cada muestra considera el valor de referencia $\left(\mathrm{PP}_{0}\right)$. Por lo tanto, se calcula I.D.T $\left(\mathrm{PP}_{\mathrm{n}}\right)=\mathrm{T}_{5}\left(\mathrm{PP}_{\mathrm{n}}\right) / \mathrm{T}_{5}\left(\mathrm{PP}_{0}\right) * 100$, donde $n=0-5$.

\section{FLUIDEZ, VISCOSIDAD Y DENSIDAD}

El índice de fluidez se determinó para el material particulado de cada generación de PP usando el Plastómetro de extrusión ATLAS MFI a $230^{\circ} \mathrm{C}, 2.16 \mathrm{~kg}$ mediante 4 cortes por cada ensayo. La viscosidad se determinó mediante un reómetro rotacional (DHR-2, TA Instrument) con esfuerzo controlado y configuración de platos paralelos utilizando el ensayo de flujo de equilibrio. Las me-

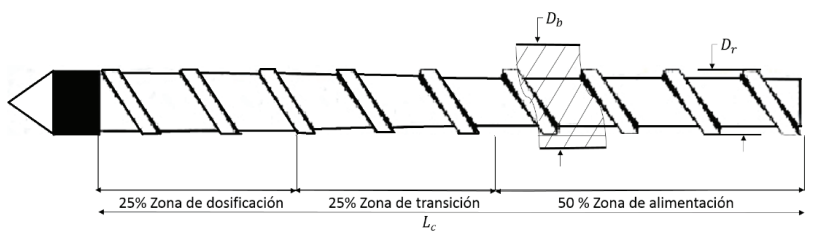

Figura 2. Tornillo de inyección estándar 
didas reológicas se realizaron a $190^{\circ} \mathrm{C}$ y la velocidad de

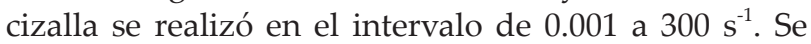
efectuaron ensayos dinamomecánicos, fijando el porcentaje de deformación en $10 \%$ con un rango de frecuencias entre 0.1 a $628.10 \mathrm{rad} / \mathrm{s}$ para determinar el módulo de almacenamiento y el módulo de pérdida. Por otro lado, la densidad y peso específico para cada generación de PP se determinó bajo instructivo de ensayo teniendo como referencia la norma NTC: 907 (método B) versión 1996-08-21 utilizando como liquido de inmersión Iso-Octano, un termómetro ASTM $23^{\circ} \mathrm{C}$ : Marca BRAND, Modelo GOLD BRAND, Rango: $8^{\circ} \mathrm{C}$ a $36^{\circ} \mathrm{C}$ con una Resolución de $0.2^{\circ} \mathrm{C}$. Un picnómetro con un rango de $23 \mathrm{~cm}^{3}$ y una balanza analítica con un rango de $0.01 \mathrm{~g}$ a $220 \mathrm{~g}$.

\section{Propiedades MeCÁNICAS}

RESISTENCIA A LA TRACCIÓN Y FLEXIÓN

Las mediciones de las propiedades mecánicas de tracción se realizaron en una maquina universal GOODBRAND de acuerdo con la norma ASTM D638, utilizando una velocidad de ensayo de $50 \mathrm{~mm} / \mathrm{min}$ y empleando una celda de $500 \mathrm{kgf}$. Se determinaron los valores de alargamiento a la rotura y resistencia a la tracción. Se empleó una cámara ambiental DIES, un calibrador pie de rey Baker y termohigrómetro OAKTON. Los ensayos de flexión se realizaron en una maquina universal INSTRON 5500R de acuerdo con la norma ASTM D790-10 utilizando una velocidad de ensayo de $5 \mathrm{~mm} / \mathrm{min}$ y celda de $50 \mathrm{kgf}$. Se obtuvieron los módulos, resistencias y porcentaje de elongación. Se analizaron cinco muestras para cada generación de PP y se presentan los valores medios.

Las muestras se acondicionaron a $25^{\circ} \mathrm{C}$ en un ambiente de humedad relativa de $50 \pm 5 \%$ durante $48 \mathrm{~h}$.

\section{Resultados}

\section{ANÁLISIS TERMOGRAVIMÉTRICO Y DSC}

Las propiedades térmicas de los productos de PP después de cada proceso se determinaron por termogravimetria (TGA) y calorimetría diferencial de barrido (DSC) los resultados se muestran en la tabla 1. Todas las generaciones presentaron una buena estabilidad térmica con valores de temperatura de degradación a 5\% de pérdida en peso $\left(T_{5}\right)$ entre $380^{\circ} \mathrm{C}$ y $330^{\circ} \mathrm{C}$. Al comparar los valores experimentales de $\mathrm{T}_{5}$ obtenidos, se observa un comportamiento térmico degradativo tipo exponencial a medida que aumenta el número de carre- ras del proceso. El análisis térmico presenta una pérdida significativa de $13.6 \%$ para la quinta generación $\left(\mathrm{PP}_{5}\right)$ respecto al material virgen $\left(\mathrm{PP}_{0}\right)$. Las temperaturas de fusión $\left(\mathrm{T}_{\mathrm{m}}\right)$ mostradas en la tabla 1 de las diferentes generaciones de PP presentaron una disminución total de $2.6^{\circ} \mathrm{C}$ para $\mathrm{PP}_{5}$ respecto a $\mathrm{PP}_{0}$. Adicionalmente, se observó la presencia de una mesofase en el $\mathrm{PP}_{1}$ antes del punto de fusión, debido a la presencia de dominios cristalinos. Lo anterior indica que los diferentes tratamientos termomecánicos que genera el reproceso favorece la obtención de estructuras amorfas.

Tabla 1. Resumen de las propiedades térmicas del PP transformado por inyección

\begin{tabular}{ccc}
\hline $\begin{array}{c}\text { Generaciones } \\
\mathrm{PP}\end{array}$ & \multicolumn{2}{c}{ Proceso de inyección } \\
$\mathrm{TP}_{5}\left({ }^{\circ} \mathrm{C}\right)$ & $\mathrm{T}_{\mathrm{m}}\left({ }^{\circ} \mathrm{C}\right)$ \\
\hline $\mathrm{PP}_{1}$ & 386.9 & 149.1 \\
$\mathrm{PP}_{2}$ & 380.5 & 147.5 \\
$\mathrm{PP}_{3}$ & 377.0 & 147.5 \\
$\mathrm{PP}_{4}$ & 369.5 & 147.5 \\
$\mathrm{PP}_{5}$ & 360.0 & 146.5 \\
\hline $\mathrm{SICIÓ}$ & 334.1 & 146.5 \\
\hline
\end{tabular}

SIMULACIÓN TÉRMICA

Se estimó el tiempo de residencia $\left(t_{r}\right)$ con los datos determinados en el experimento de inyección, que relacionan la ficha técnica del equipo y material polimérico utilizado: $V_{t}=245.5 \mathrm{~cm}^{3}, \rho_{m}=0.73 \frac{\mathrm{g}}{\mathrm{cm}^{3}}, t c=28 \mathrm{~s} \mathrm{y}$ $w_{i}=23.8 \mathrm{~g}$.

$t_{r}(\min )=\frac{\left(0.73 \frac{\mathrm{g}}{\mathrm{cm}^{3}}\right)\left(245.5 \mathrm{~cm}^{3}\right)(28 \mathrm{~s})}{(23.8 \mathrm{~g})(60 \mathrm{~s})}=3.51 \mathrm{~min}$

Es importante mencionar que el tiempo residencia obtenido no considera el volumen adicional $(40 \%)$ a la capacidad máxima del tornillo, sugerida por el autor Dilán (2002).

Las generaciones obtenidas por simulación térmica se sometieron a las siguientes condiciones: temperatura máxima de proceso $240^{\circ} \mathrm{C}$, tiempo de residencia $\left(t_{r}\right) 3.51$ min, se ingresa el material al horno con condiciones descritas. Posteriormente, se deja en reposo a temperatura ambiente y se repite el proceso a cada muestra hasta alcanzar los cinco ciclos.

En la tabla 2 se muestran los resultados del análisis termogravimétrico de las muestras $\mathrm{PP}_{0 s^{\prime}} \mathrm{PP}_{1 s^{\prime}} \mathrm{PP}_{3 \mathrm{~s}}$ y $\mathrm{PP}_{5 \mathrm{~s}}$ sometidas a ciclos de calentamiento. Los termogramas 
mostraron una pérdida total de $3.8 \%$ para $\mathrm{PP}_{5 \mathrm{~s}}$ respecto a $\mathrm{PP}_{0 \mathrm{~s}}$.

En la figura 3, se observa el índice de desempeño térmico que considera el $\mathrm{T}_{5}$ para cada proceso que sufrió el material polimérico. La línea punteada presenta una tendencia lineal de degradación para las cinco generaciones del proceso de calentamiento. Mientras que la línea solida muestra una tendencia exponencial del proceso de inyección como producto de los drásticos esfuerzos mecánicos que afronta el material.

El índice de fluidez para las diferentes generaciones se presenta en la figura 4, este material mostró un incremento de $155.4 \%$ en la quinta generación respecto al material virgen. Este aumento progresivo representa degradación en el material asociado con la disminución del peso molecular, que se calculó indirectamente con la determinación de la viscosidad intrínseca para las muestras $\mathrm{PP}_{0}, \mathrm{PP}_{1}, \mathrm{PP}_{3}$ y $\mathrm{PP}_{5}$ que muestran una reducción en los valores iniciales descritos en la curva reológica a bajas velocidades de cizalladura, donde se observa el Plateau Newtoniano $\left(\eta_{0}\right)$ (figura 5). La viscosidad de cizalla es una propiedad importante en polímeros fundidos, ya que es directamente proporcional a la masa molar promedio.

Por otro lado, se calculó el índice de polidispersidad (IP) mediante la determinación del Módulo de almacenamiento $G^{\prime}$ y Módulo de pérdida $G^{\prime \prime}$ en función de la frecuencia angular para las muestras $\mathrm{PP}_{0}$ y $\mathrm{PP}_{5}$. Los valores asociados a los puntos donde se cortan ambos módulos ( $G^{\prime}$ y $G^{\prime \prime}$ ) se representa como $G_{c}$ (figura 6), este valor se utiliza en la siguiente ecuación (Shroff and Mavridis, 1995): $\mathrm{IP}=1 \mathrm{E} 5 / \mathrm{G}_{\mathrm{c}}$

Los valores de IP para $\mathrm{PP}_{0}$ y $\mathrm{PP}_{5}$ fueron de $2.99 \mathrm{y}$ 3.05 , respectivamente. Estos valores crecientes de IP in-

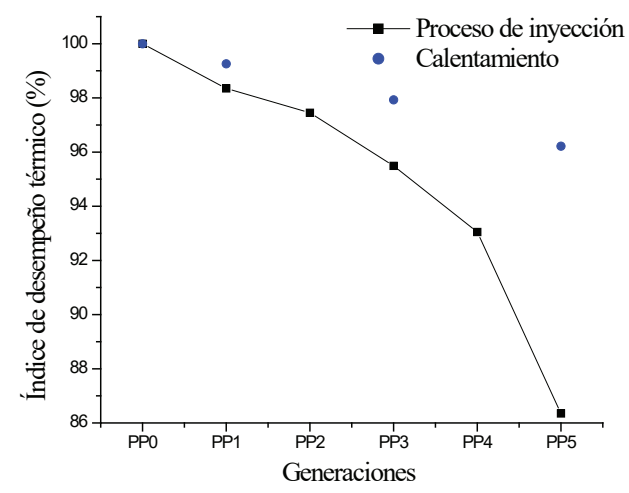

Figura 3. Índice de desempeño para los procesos térmicos dican ampliación de la distribución de peso molecular (DPM) en general.

Por último, se realizó el ensayo físico de densidad donde se obtuvo un índice de pérdida de $4.1 \%$ para $\mathrm{PP}_{5}$, comparado con el material $\mathrm{PP}_{\mathrm{o}^{\prime}}$ lo cual no se muestra significativo para el proceso de inyección por factores como la temperatura y la fricción. Los valores se presentan a continuación en la figura 7 .

\section{Propiedades mecánicas}

Con el fin de conocer la influencia del reproceso para el material PP sobre las propiedades mecánicas se evaluó la resistencia a la flexión y módulo de Young mediante el ensayo de Flexión como se observa en la figura 8. Para este ensayo la muestra con mayor reprocesos $\left(\mathrm{PP}_{5}\right)$ presentó un índice de pérdida de Flexión de 6.0\%, lo cual indica que los reprocesos en el material PP por inyección no afectan considerablemente esta propiedad. Mientras que el índice de pérdida del módulo de Young para la misma muestra reveló un índice de pérdida de

Tabla 2. Resumen de las propiedades térmicas del PP: rampa de calentamiento

\begin{tabular}{ccc}
\hline \multirow{2}{*}{$\begin{array}{c}\text { Generaciones } \\
\mathrm{PP}\end{array}$} & \multicolumn{2}{c}{ Calentamiento } \\
\hline $\mathrm{T}_{5}\left({ }^{\circ} \mathrm{C}\right)$ & $\mathrm{T}_{\mathrm{m}}\left({ }^{\circ} \mathrm{C}\right)$ \\
\hline $\mathrm{PP}_{1 \mathrm{~s}}$ & 386.90 & 149.1 \\
$\mathrm{PP}_{3 \mathrm{~s}}$ & 384.04 & 149.0 \\
$\mathrm{PP}_{5 \mathrm{~s}}$ & 378.89 & 148.7 \\
\hline${ }^{a}$ Velocidad de calentamiento & $10^{\circ} \mathrm{C} / \mathrm{min}$
\end{tabular}

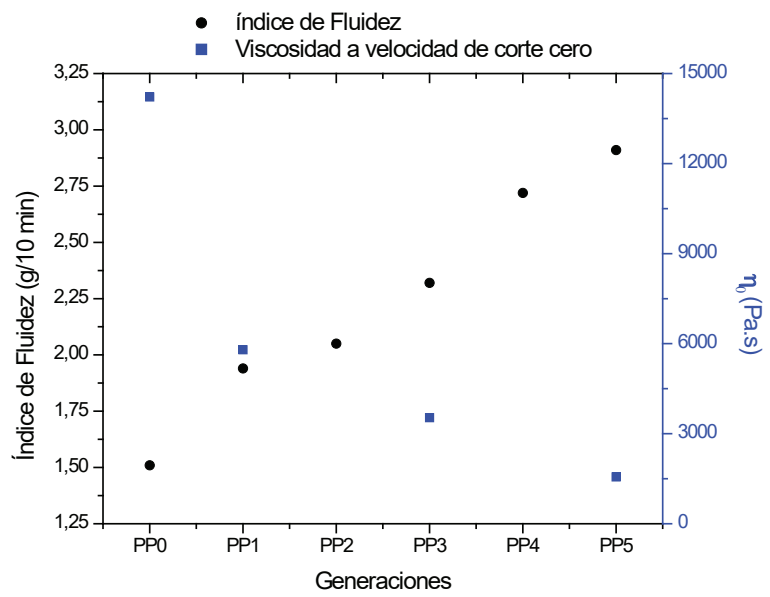

Figura 4. Resultados de índice de fluidez (Y1) y viscosidad intrínseca (Y2) para PP reprocesado 
9.0\% comparado con el material inyectado una sola vez $\left(\mathrm{PP}_{1}\right)$.

Por otro lado, se determinó la resistencia a la tensión y elongación máxima como se puede ver en la figura 9. El análisis correspondiente al ensayo de tracción presentó un índice de desempeño de pérdida de $8.4 \%$ para el último reproceso $\left(\mathrm{PP}_{5}\right)$.

\section{ConClusiones}

En este trabajo se llevó a cabo el reproceso del material polimérico PP mediante la técnica de inyección, el cual

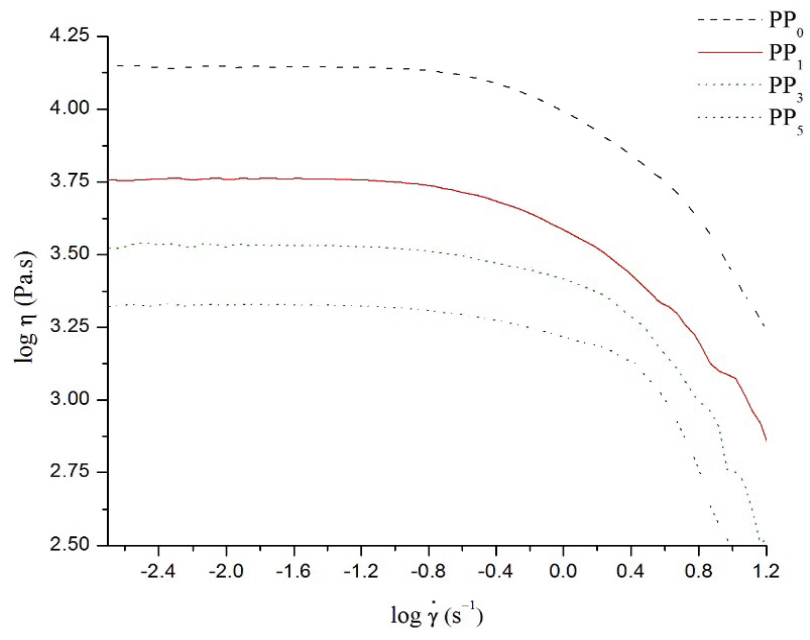

Figura 5. Resultados logarítmicos de la viscosidad en función de la velocidad de corte de las diferentes generaciones de PP

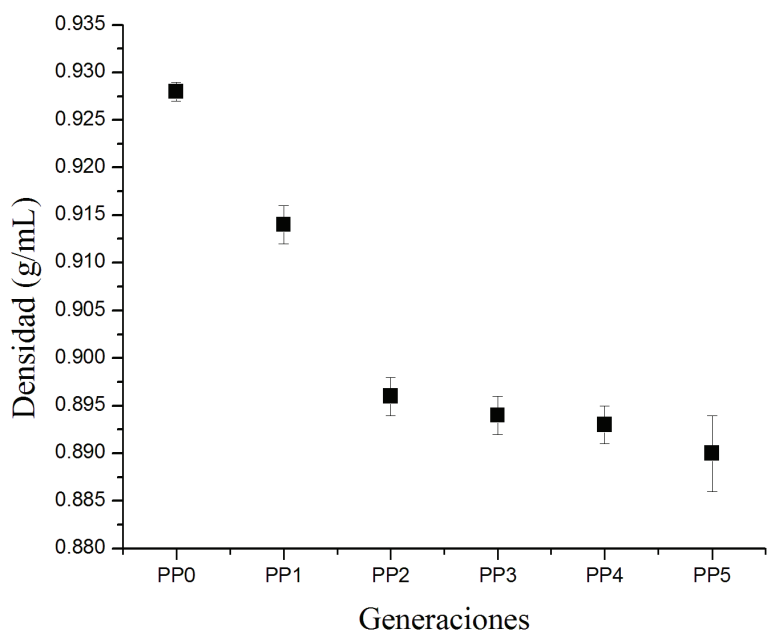

Figura 7. Resultados de densidad para PP reprocesado sugirió un ajuste en los parámetros de proceso que se utilizó a través de las generaciones para la obtención de las probetas. Estas se caracterizaron térmicamente por TGA y DSC, los resultados mostraron una clara degradación del material al aumentar los ciclos de proceso, este hecho se logró corroborar con los resultados obtenidos de los ensayos de índice de fluidez, viscosidad y densidad, los cuales permitieron identificar de forma indirecta la disminución en el peso molecular y la tendencia que presentan las cadenas poliméricas cortas a fluir progresivamente en el material reprocesado. El efecto de los esfuerzos de corte y compresión asociado

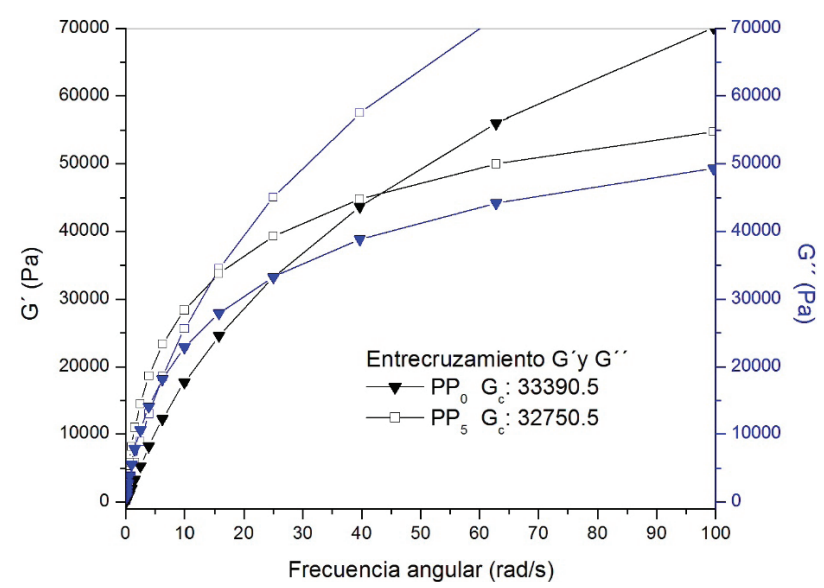

Figura 6. Comparación de los barridos de frecuencia para $\mathrm{PP}_{0} \mathrm{y}$ $\mathrm{PP}_{5}$

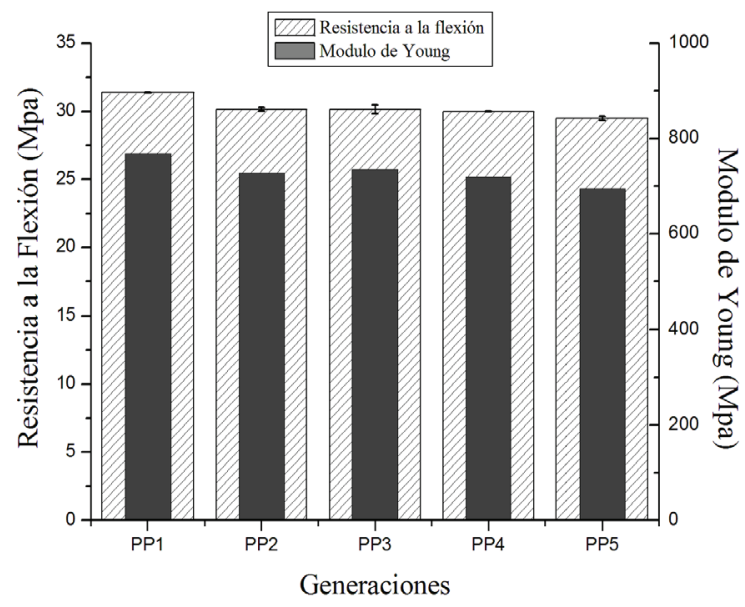

Figura 8. Resistencia a la flexión de las generaciones de PP 


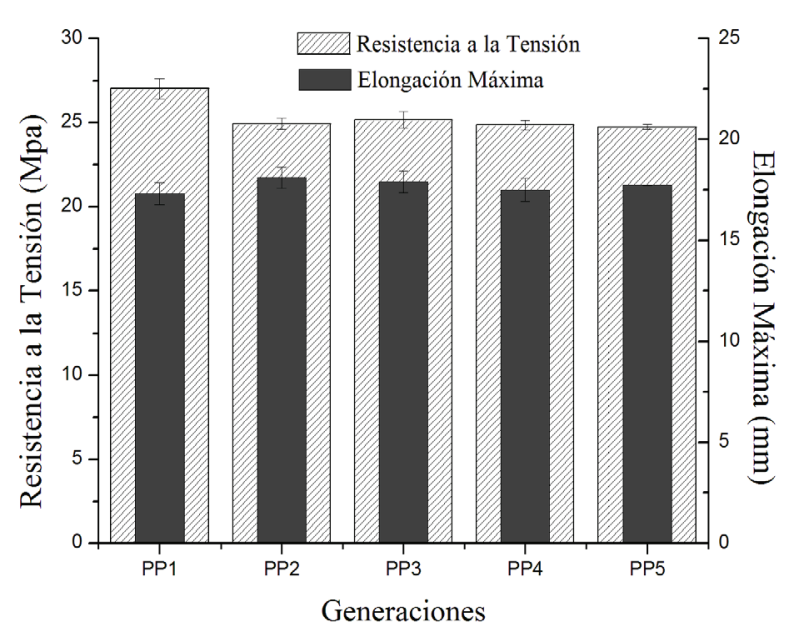

Figura 9. Resistencia a la tracción de las generaciones de PP

al proceso de transformación del material presentó una degradación térmica de $9.9 \%$ comparada con el proceso de calentamiento, este se calculó como la diferencia entre puntos para la quinta generación detallada en la gráfica del índice de desempeño térmico. Adicionalmente, los resultados de $T_{5}$ de la quinta generación se mantienen por encima de la temperatura de proceso, lo cual abre camino para realizar un análisis generacional más alto. Este trabajo ratifica las tendencias enmarcadas sobre degradación de polímeros con datos precisos, lo que genera de forma positiva que se promueva el uso de material reprocesado de PP como valor agregado, sobre todo a la industria automotriz. Asimismo, este trabajo constituye un antecedente para promover el desarrollo de mezclas con material reprocesado, garantizando que persista inalterado en propiedades térmicas y mecánicas dentro de un nuevo producto.

\section{Agradecimientos}

Este trabajo se desarrolló gracias al apoyo del Centro Astin, SENA y el grupo de investigación en desarrollo de materiales y productos (GIDEMP), así como al Departamento de Biotecnología del Tecnoparque Nodo Cali. C.C. agradece el soporte económico del contrato de financiamiento de recuperación contingente Núm. FP44842-259-2015, COLCIENCIAS-SENA.

\section{ReferenCiAS}

Bahlouli N., Pessey D., Raveyre C., Guillet J., Ahzi S., Dahoun A., Hiver J.M. Recycling effects on the rheological and thermomechanical properties of polypropylene-based composites. Materials and Design, volumen 33, 2012: 451-458.
Bledzki A.K., Franciszczak P., Osman Z., Elbadawi M. Polypropylene biocomposites reinforced with softwood, abaca, jute, and kenaf fibers. Industrial Crops and Products, volumen 70, 2015: 91-99.

Brenner E. Polypropylene an alternative? Kunststoffe, volumen 4, 2000: 35.

Da Costa H.M., Ramos V.D., De Oliveira M.G. Degradation of polypropylene (PP) during multiple extrusions: Thermal analysis, mechanical properties and analysis of variance. Polymer Testing, volumen 26, 2007: 676-684.

Dilán H. Técnicas modernas de moldeo. Moldeo Universal ${ }^{\mathrm{TM}}$. Copyright Hector Dilán, 2002.

Gonzalez J.G, Neira-Velazquez G., Angulo-Sanchez J.L. Polypropylene chain scissions and molecular weight changes in multiple extrusion. Polym Degrad Stab, volumen 60 (número 1), 1998: 33.

Harutun K. Handbook of Polypropylene and Polypropylene composites, $2^{a}$ ed., CRC Press, 2003, pp. 10-29.

Hermanová S., Tochácek J., Jancár J., Kalfus J. Effect of multiple extrusion on molecular structure of polypropylene impact copolymer. Polymer Degradation and Stability, volumen 94, 2009: 1722-1727.

Hoffman J. Polyprpylene beginning to show signs of strength. Chemical market Reporter, 2002: 8.

Kang H., Lu X., Xu Y. Properties of immiscible and ethylene-butyl acrylate-glycidyl methacrylate terpolymer compatibilized poly (lactic acid) and polypropylene blends. Polymer Testing, volumen 43, 2015: 173-181.

Market Study, Polypropylene, $3^{\mathrm{a}}$ ed., Ceresana, 2013.

Muccio E.A. Plastics Processing Technology, 1994: 152-153.

Papageorgioua D.G., Bikiaris N., Chrissafis K. Effect of crystalline structure of polypropylene random copolymers on mechanical properties and thermal degradation kinetics. Thermochimica Acta, volumen 543, 2012: 288-294.

Shroff R. y Mavridis H. J. Appl. Polym. Sci., volumen 57, 1995: 1605-1626.

Thakur V.K., Vennerberg D., Kessler M.R. Green aqueous surface modification of polypropylene for novel polymer nanocomposites. ACS Appl. Mater. Interfaces, volumen 6 (número 12), 2014: 9349-9356. 


\section{Citación sugerida}

\section{Citación estilo Chicago}

Caicedo, Carolina, Lina Marcela Crespo-Delgado, Hever De La CruzRodríguez, Norman Andrés Álvarez-Jaramillo. Propiedades termomecánicas del Polipropileno: Efectos durante el reprocesamiento. Ingeniería Investigación y Tecnología, XVIII, 03 (2017): 245-252.

\section{Citación estilo ISO 690}

Caicedo C., Crespo-Delgado L.M., De La Cruz-Rodríguez H., ÁlvarezJaramillo N.A Propiedades termo-mecánicas del Polipropileno: Efectos durante el reprocesamiento. Ingeniería Investigación y Tecnología, volumen XVIII (número 3), julio-septiembre 2017: 245-252.

\section{SemblanZas de los autores}

Carolina Caicedo: Química de la Universidad del Quindío, Colombia (2001-2006), realizó su maestría y doctorado en ciencia e ingeniería de materiales en el Instituto de Investigaciones en Materiales de la Universidad Nacional Autónoma de México (2007-2013). Realizó una estancia posdoctoral en el grupo de física y química teórica de la Facultad de Química de la UNAM (2013-2015). Actualmente es investigadora en el Centro Nacional de Asistencia Técnica a la Industria (ASTIN) del SENA en convenio con COLCIENCIAS, Colombia (2015-2017).

Lina Marcela Crespo-Delgado: Ingeniera de materiales, magister en ingeniería con énfasis en ingeniería de materiales por la Universidad del Valle. Es investigadora junior en COLCIENCIAS. Docente e investigadora en el Grupo de Investigación en Desarrollo de Materiales y Productos (GIDEMP) del Centro de Asistencia Técnica para la Industria (ASTIN), SENA.

Hever De la Cruz-Rodríguez: Ingeniero mecatrónico por la Universidad Autónoma de Occidente, es instructor técnico en el área de inyección de plásticos en el centro ASTIN del SENA, es docente e investigador en el Grupo de Investigación en Desarrollo de Materiales y Productos (GIDEMP) del Centro ASTIN. Cuenta con 20 años de experiencia en el área de formación profesional y asistencia técnica en empresas del sector del plástico en Colombia.

Norman Andrés Álvarez-Jaramillo: Ingeniero de materiales por la Universidad del Valle. Participa activamente en el programa tecnológico para la fabricación de productos plásticos por inyección y soplado en el Centro ASTIN-SENA y en el grupo de semilleros del Centro ASTIN. Es monitor de apoyo a docencia en el Centro ASTIN. 